\title{
ESE-1 Knockdown Attenuates Growth in Trastuzumab-resistant HER2+ Breast Cancer Cells
}

\author{
ADWITIYA KAR ${ }^{1}$, BOLIN LIU ${ }^{2}$ and ARTHUR GUTIERREZ-HARTMANN ${ }^{1,3,4}$ \\ ${ }^{1}$ Cancer Biology Graduate Program, ${ }^{2}$ Department of Pathology, ${ }^{3}$ Department of Medicine, \\ ${ }^{4}$ Department Biochemistry \& Molecular Genetics, \\ University of Colorado Anschutz Medical Campus, Aurora, CO, U.S.A.
}

\begin{abstract}
Background/Aim: ESE-1/Elf3 controls transformation properties in mammary epithelial cells, and is most clinically relevant in $\mathrm{HER}_{2}{ }^{+}$breast cancer. Herein we showed that ESE-1 knockdown inhibits tumorigenic growth in $H E R 2^{+}$, trastuzumabresistant HR2O (derived from $\mathrm{HER}^{+} E \mathrm{R}^{+}$BT474) and Pool2 (derived from $H E R 2^{+} E R-S K B R 3$ cells) cell lines. Materials and Methods: We used cell proliferation, clonogenicity, viability, and soft agar assays to measure the effects of ESE-1 knockdown in cell lines. Results: ESE-1 knockdown in the resistant cell lines inhibited HER 2 and other downstream effectors in a cell-type specific manner, but caused down-regulation of pAkt and cyclin D1 in both sublines. In parental BT474 and SKBR3 ESE-1 silencing revealed a potent anti-proliferative effect that mimics the trastuzumab-mediated growth inhibition but did not enhance trastuzumab sensitivity in the resistant sublines. Conclusion: This study provides rationale to study ESE-1 as a novel mean to treat $H E R 2^{+}$patients who show resistance to anti-HER2 therapy.
\end{abstract}

Trastuzumab, also known as Herceptin, is a monoclonal antibody that interrupts HER2-mediated downstream signaling by various mechanisms (1-3). In combination with chemotherapy, trastuzumab has been shown to increase overall survival in HER $2^{+}$breast cancer patients (4). However, only $30 \%$ of all HER $2^{+}$breast cancer patients respond to trastuzumab, and often duration of response to trastuzumab lasts only 5 to 9 months, indicating that both primary and acquired resistance to trastuzumab is common. Combination therapies with lapatinib and gefitinib have also been fraught

This Article is freely accessible online.

Correspondence to: Arthur Gutierrez-Hartmann, Departments of Medicine and of Biochemistry \& Molecular Genetics, University of Colorado Anschutz Medical Campus, Mail Stop 8106, Aurora, CO 80045, U.S.A. Tel: +1 3037243921, Fax: +1 3037243920, e-mail: a.gutierrez-hartmann@UCDenver.edu

Key Words: ETS, Elf3, Her2 signaling, cyclin D1, trastuzumab. with resistance arising from compensatory signaling molecules or pathways $(1,5)$. There is, therefore, a need for studying other effectors or modulators that influence the durability of response and/or by-pass resistance to HER2-targeted therapies.

Several ETS transcription factors, such as ETS-1, ESE-1/Elf3, ESE-2/Elf5 and PEA-3, appear to be important in human breast cancer. ESE-1 is particularly relevant in HER2 ${ }^{+}$breast cancer, because ESE- 1 regulates the HER $2^{+}$promoter activity and protein levels (6). In parental BT474 and SKBR3 cells, ESE-1 controls HER2 dependent signaling and tumorigenesis (7). Moreover, neuregulin and other growth factor ligands induce ESE-1 expression (8), revealing an additional feedforward level of control of ESE-1 and downstream effectors targeted by trastuzumab.

In this paper, we investigated the role of ESE-1 in controlling transformation in trastuzumab-resistant HER2 ${ }^{-}$ positive cell lines derived from parental HER2 ${ }^{+}, \mathrm{ER}^{+} \mathrm{BT} 474$ and HER2 ${ }^{+}, \mathrm{ER}^{-}$SKBR3 breast cancer cell lines. These trastuzumab-resistant cell lines develop an interaction between HER2, HER3/ErbB3 and IGF-1R to form a hetero-trimeric receptor signaling complex as the mechanism mediating trastuzumab resistance (9). Investigating ESE-1's role in the context of trastuzumab-resistance showed that knockdown of ESE-1 inhibits proliferation, clonogenicity and anchorageindependent growth in both resistant cell lines specifically by modulating several signaling molecules. Furthermore, lack of any synergistic inhibitory response of trastuzumab plus ESE1 knockdown in the parental lines revealed that HER2 and ESE-1 function in the same pathway. Taken together, these studies highlight ESE-1 as a by-pass effector in HER2 resistance and establish the utility of pursuing ESE-1 as a future therapeutic target to inhibit the counter-regulatory responses to trastuzumab-mediated tumor inhibition.

\section{Materials and Methods}

Cell lines and cell culture. Cell lines BT474 and SKBR3 were purchased from the tissue culture core at University of Colorado Anschutz Medical Campus and the trastuzumab resistant cell lines HR20 and Pool 2 were provided by Dr. Bolin Liu. All cell lines were 
maintained in DMEM/F12 Ham in presence of $10 \%$ FBS Trastuzumab resistant cell lines were cultured under the continuous presence of $20 \mu \mathrm{g} / \mathrm{ml}$ of trastuzumab.

shRNA transduction. The pLKO.1 shRNA construct targeting ESE1 was purchased from Open Biosystems (Lafayette, CO, USA). Oligonucleotide shESE-1 (5'-gccatgaggtactactacaaac-3') targets the ETS domain. The shScr is a scramble sequence control and was a gift from Dr. Bolin Liu (9). shRNAs were packaged and transduced following methods described previously (7). All experiments were plated eight days post transduction and long-term stable knockdown clones of ESE-1 were not selected to avoid a counter-selective response to cell growth inhibition mediated by ESE-1 knockdown.

Western blotting. Cells were lysed in RIPA buffer and immunoblotting was performed using procedures described previously (7). Immunoblotting for ESE-1 was performed using anti-ESE-1 monoclonal antibody (mAb405) developed in our lab (10). Antibodies against IGFR, ERBB3, mTOR, Src, HER2, Akt, MAPK were purchased from Cell signaling (Danvers, MA, USA) and were used following manufacturer's protocol at a dilution of 1:1,000.

MTS assay. MTS assay (Promega, Madison, WI, USA) was performed according to manufacturer's protocol. Briefly,5000 cells were plated, then at 3 days or 4 days post-plating 1X MTS assay buffer was added to the cells, and cells were allowed to incubate at room temperature for $10 \mathrm{~min}$ with $2 \mathrm{~min}$ of additional shaking to allow for cell lysis. Samples were then measured using the luminescence setting using an HT Synergy plate reader.

Proliferation, clonogenicity and soft agar assays. For cell proliferation assays, cells were transduced with shRNA and plated in duplicates in $10 \mathrm{~cm}$ plates and viable cells were counted by Vicell on days 1, 3, 5 and 7. For clonogenicity studies, 3,000 cells per well were plated in quadruplicate in 6-well plates. For soft agar assays, transduced Pool2 and HR20 cells were suspended at 30,000 cells/well and 20,000 cells/well, respectively, and plated in 6-well plates. Quantification of colonies were done using previously described methods (7).

\section{Results}

ESE-1 knockdown inhibits proliferation, clonogenicity and anchorage-independent colony growth in trastuzumabresistant HER2 ${ }^{+}$BT474 and SKBR3 breast cancer cell lines. Trastuzumab-resistant cells HR20 and Pool2 cell lines, which were derived from parental HER2 ${ }^{+}$BT474 and SKBR3 cells, respectively, by continuous exposure to trastuzumab $(11,12)$ was used to determine if ESE-1 controls tumorigenic properties. These cells have been maintained under the continuous presence of $20 \mu \mathrm{g} / \mathrm{ml}$ of trastuzumab, a condition in which parental BT474 and SKBR3 cells do not survive. ESE-1 KD resulted in a near complete inhibition of ESE-1 protein in HR20 (Figure 1A) and about a $60 \%$ decrease of ESE-1 in Pool2 (Figure 1B). In the cell proliferation assay, viable cells were counted from days 1 to day 7 in the scrambled (scr) control and the KD group. ESE-1 knockdown inhibited cell number at each of the 7 days in both HR20 and
Pool 2 cell lines, with the reduction in HR20 being more pronounced, with an $84 \%$ decrease in proliferation (Figure 1A) and Pool 2 cells showing a 54\% inhibition in cell number, each compared to the scramble control at day 7 (Figure 1B).

To assess clonogenicity, an equal number of viable shScr and shESE-1 HR20 and Pool 2 cells were plated at low density in complete media. ESE-1 KD in HR20 cells resulted in a $50 \%$ reduction in $2 \mathrm{D}$ colony formation $(p=0.0002)$ (Figure 1C) and a 70\% reduction in colony number in Pool2 cells with shESE-1 compared to shScr control $(p=0.002)$ (Figure 1D). Likewise, ESE-1 KD in HR20 cells resulted in a $62 \%$ decrease in soft agar colonies with shESE-1 $(p=0.024)$ (Figure 1E) and ESE- KD in Pool 2 cells resulted in $52 \%$ reduction $(p=0.002)$ (Figure $1 \mathrm{~F})$.

Characterization of growth factor signaling in trastuzumab treated HER2 ${ }^{+}$BT474 and SKBR3 breast cancer cell lines, and their trastuzumab resistant counterpart. As a control for the effects of trastuzumab on HER2 signaling, we first added $20 \mu \mathrm{g} / \mathrm{ml}$ of trastuzumab to the parental BT474 and SKBR3 cells, and performed Western blot analysis on multiple downstream signaling effectors (Figures 2A and 2B). In both parental lines, trastuzumab treatment down-regulated phosphorylation of HER2, Akt, as previously reported (3) (Figures 2A and 2B). However, the phosphorylation level of tyrosine kinase receptor HER3, which is considered to be the dimerizing partner of HER2, tyrosine kinase IGF-1R, and the non-receptor tyrosine kinase adaptor molecule Src, displayed cell type-specific responses to trastuzumab treatment (Figures 2A and 2B). Specifically, trastuzumab treatment inhibited HER3, IGF-IR and Src phosphorylation in BT474 cells, but induced their activation in the SKBR3 cells (Figure 2A). Trastuzumab treatment also caused a decrease in pmTOR in both cell types (Figures 2A and 2B). Because ESE1 and HER 2 are involved in a positive feedback loop, we determined whether treatment of the parental cell lines with trastuzumab inhibited ESE-1 protein levels. While trastuzumab treatment of BT474 cells resulted in a $17 \%$ inhibition of ESE-1 protein expression when normalized to tubulin (Figure 2A), ESE-1 inhibition was not observed in the trastuzumab-treated SKBR3 cells, despite a detectable down-regulation of pHER2 in these cells (Figure 2B).

We next investigated the basal activation or expression levels of HER2, HER3, Akt, ERK, and Src in the HR20 and Pool2 trastuzumab-resistant sublines compared to their parental lines and to trastuzumab-treated parental lines. Figures $2 \mathrm{~A}$ and 2B are representative blots of three independent replicates. Both HR20 and Pool 2 were grown in the constant presence of trastuzumab $(20 \mu \mathrm{g} / \mathrm{ml})$, and these cells exhibited either similar or slightly lower levels of pHER2, HER2, pAKT, pERK expression compared to their basal expression in the parental counterparts (Figure 2A and B). In terms of HER3 activation, Pool 2 cells showed high pHER3 compared to parental SKBR3, 
A

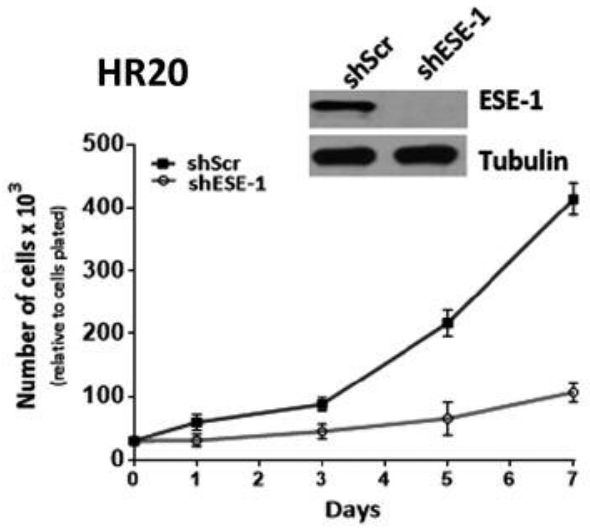

C
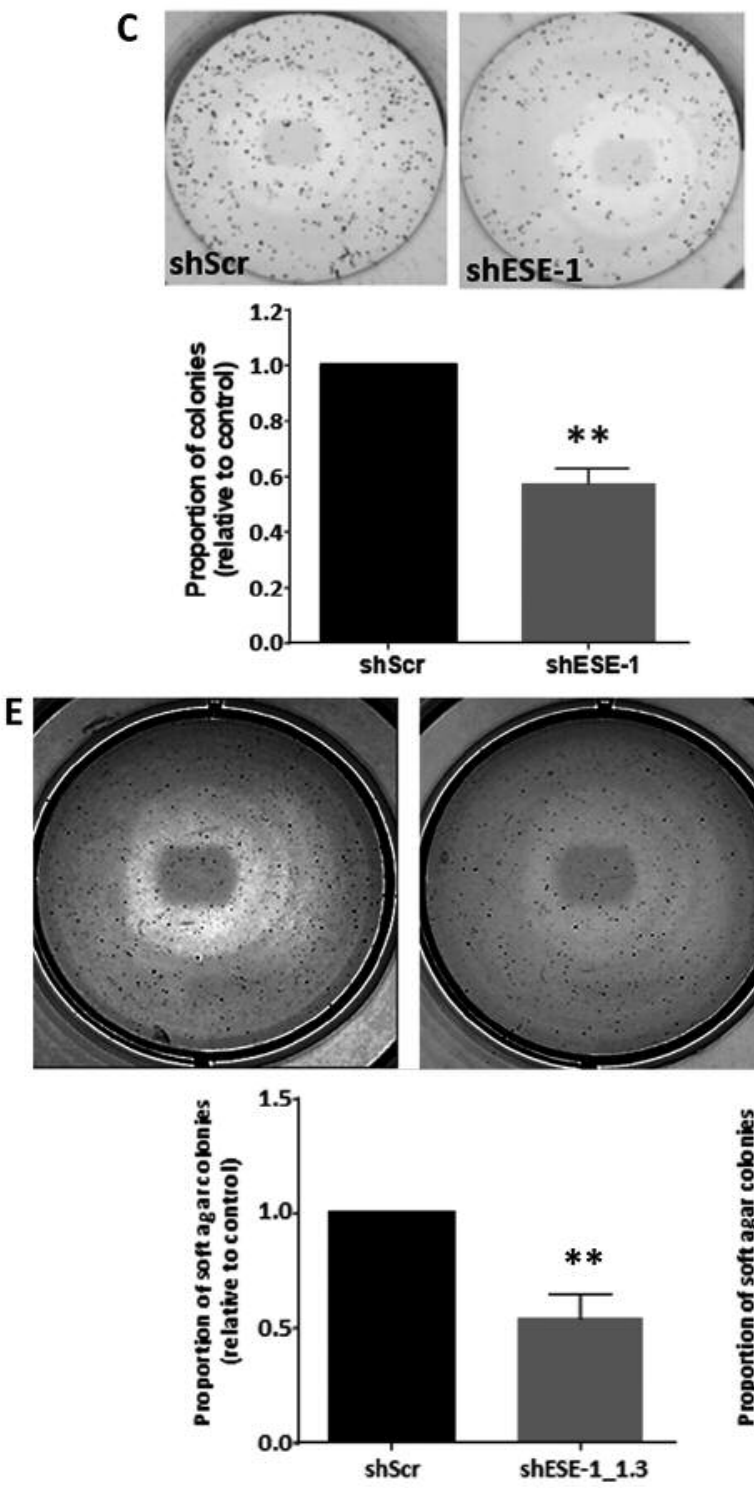

B

Pool2

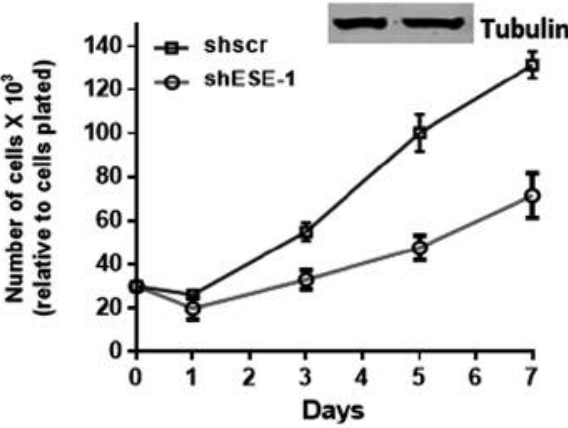

D
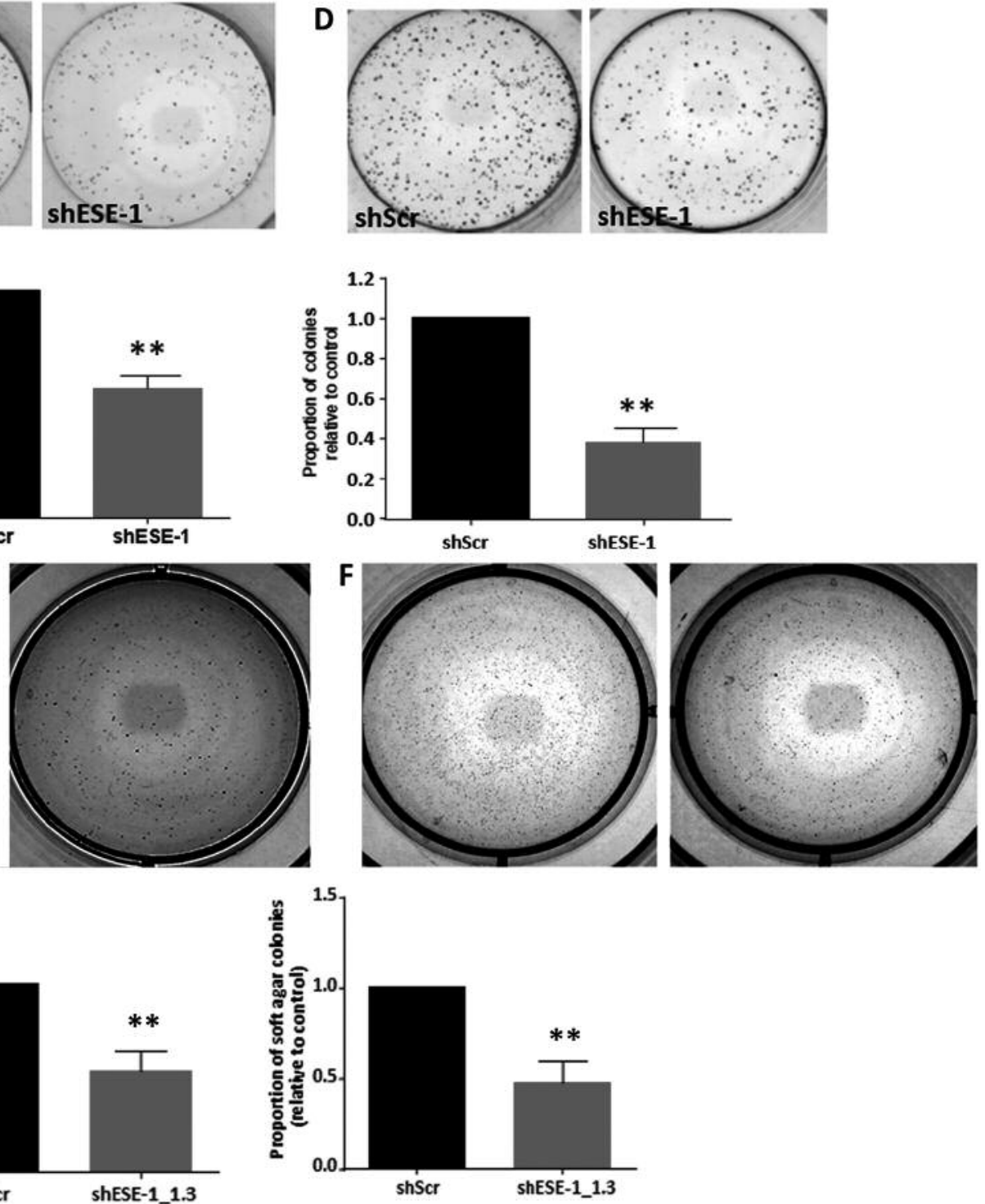

Figure 1. ESE-1 knockdown inhibits transformation properties in trastuzumab resistant HER2 ${ }^{+}$cell lines. A, B: Stable ESE-1 knockdown inhibits proliferation in trastuzumab-resistant HER2+ cell lines BT474-HR2O and SKBR3-Pool2 cell line. Proliferation was measured via the trypan blue exclusion method by Vicell. C, D, E, F: Down-regulation of ESE-1 inhibits clonogenicity, and soft agar colony formation in the two resistant sublines. Data represented is cumulative of three independent replicates. Error bars represent mean \pm SEM derived from three independent experiments with $* *$ denoting $p$-values $<0.05$. 

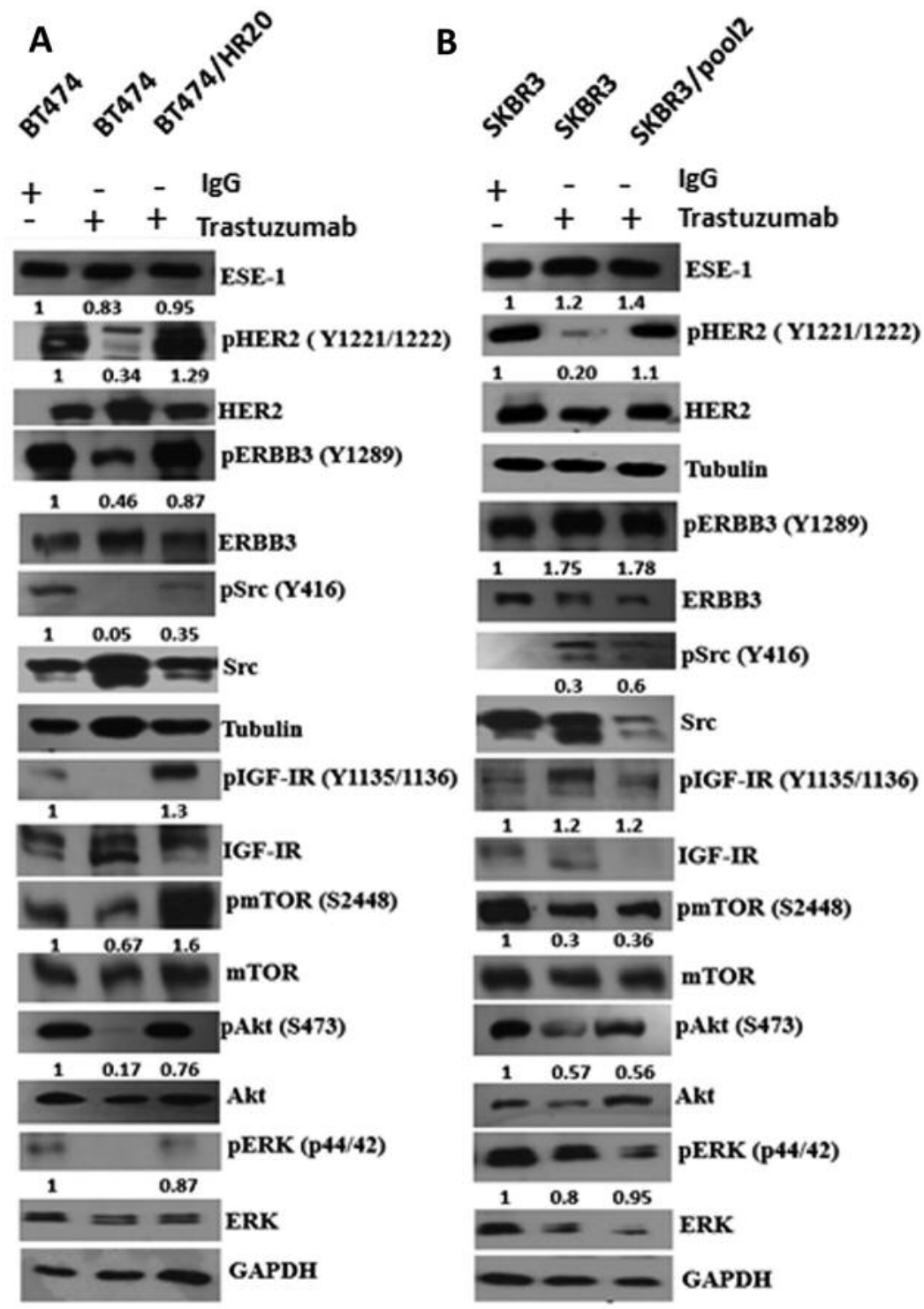

Figure 2. Immunoblot showing basal levels of signaling proteins in parental, trastuzumab-treated, and trastuzumab-resistant HER2+ cell lines. A, B: Immunoblotting was done with parental cell lines maintained in DMEM/F12 Ham, BT474 and SKBR3 cells treated with $20 \mu \mathrm{g} / \mathrm{ml}$ trastuzumab for a period of 3 and 4 days respectively, and with trastuzumb resistant HER2+ lines BT474-HR2O and SKBR3-Pool2 stably maintained in $20 \mu \mathrm{g} / \mathrm{ml}$ of trastuzumab. Quantification of the blot was done using the Image J software. All proteins were first normalized to the loading control. Phosphorylation levels were then estimated by normalizing the phosphorylation densities to their respective total.

while HR20s displayed similar levels of pHER3 as their parental cells. Both cell lines, however, exhibited increased Src phosphorylation (Figure 2A and B). Src activation in Pool2 cells was higher than in parental SKBR3 cell lines, and Src phosphorylation levels in the HR20 cells, although lower than parental BT474 cells, was higher than the trastuzumab treated BT474 cells. These results suggest that sustained Src activation, albeit at a low level, contributes to their acquiring trastuzumab resistance. HR20 cells also evinced an induction in IGF-IR and mTOR phosphorylation, indicating additional pathways are active in these cells.

ESE-1 modulates downstream pAkt and cyclin D1 protein levels to control cell growth in trastuzumab-resistant HR2O and Pool 2 sublines. We have previously established that ESE1 knockdown does not induce apoptosis in transformed breast cancer cells, but rather inhibits cell proliferation (7). Knocking-down ESE-1 in HR20 and Pool2 trastuzumab- 
HR20

A

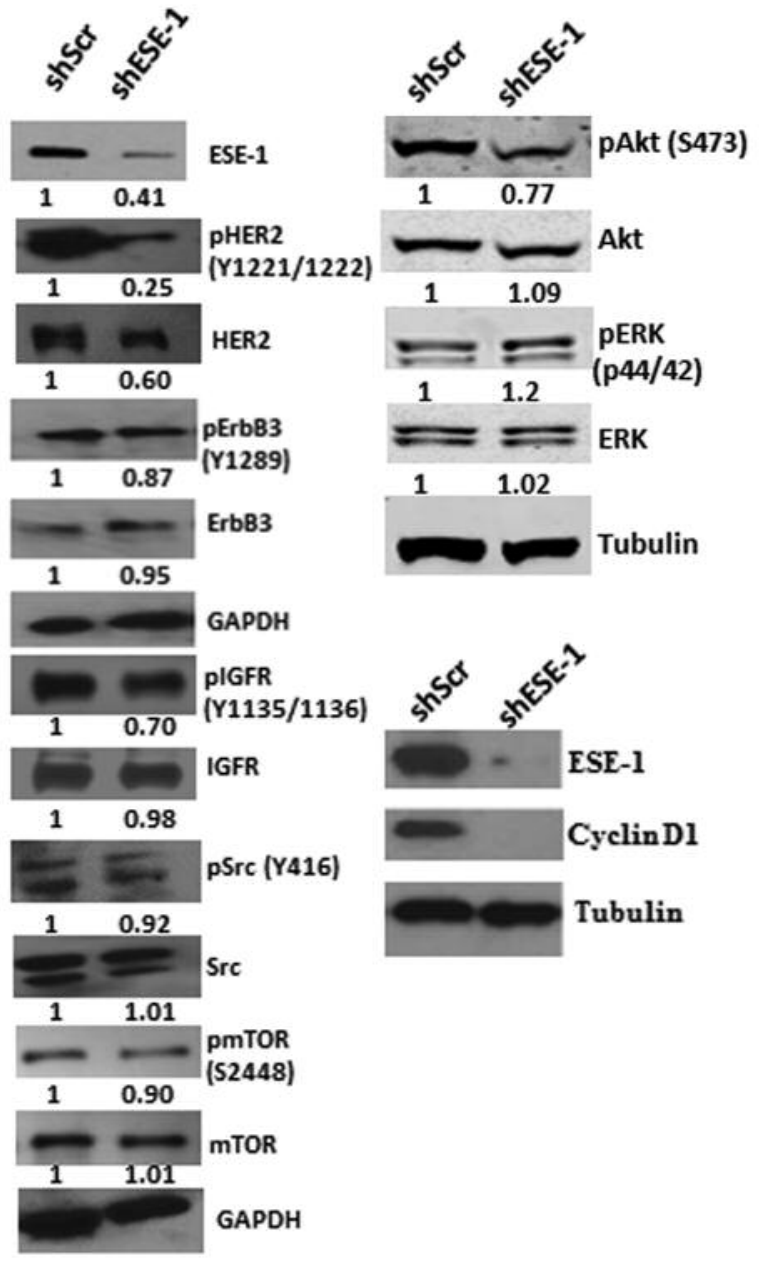

\section{Pool2}

B

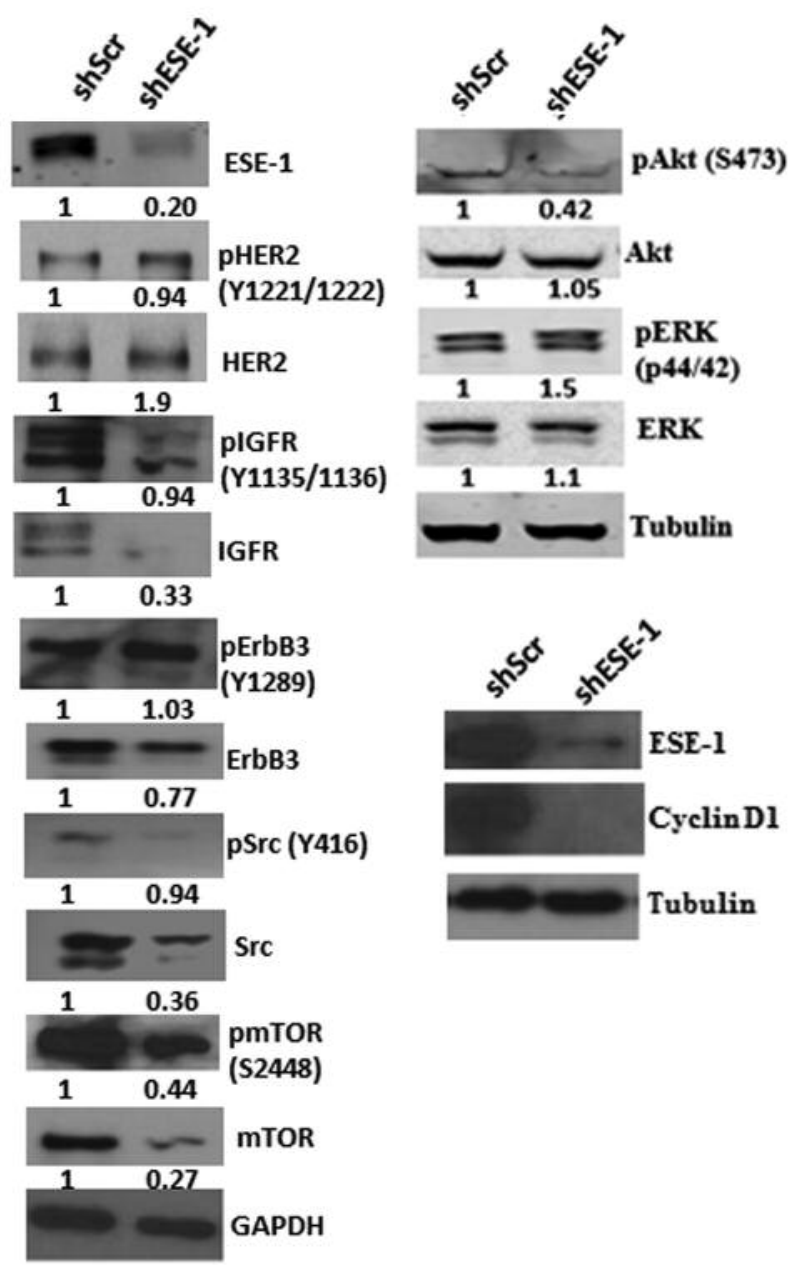

Figure 3. Immunoblot showing changes in signaling proteins in shESE-1 transduced trastuzumab-resistant BT474-HR20 and SKBR3-Pool2 cell lines. A, B: Both resistant sublines were transduced with shESE-1, was puromycin selected and was harvested in RIPA buffer 8 days post-transduction for subsequent immunoblotting. Blot shown is representative of three independent experiments. Quantification was done using the Image J software. All protein densities were first normalized to tubulin or GAPDH. Levels of phosphorylation were estimated by normalizing the phosphorylation densities to their respective total.

resistant cells inhibited tyrosine kinases and their downstream effectors in a cell type-specific manner (Figure 3A-B). ESE1 knockdown down-regulated HER2 phosphorylation and HER2 protein expression in the HR20 cell line, although HER2 activation and protein expression in Pool 2 cells were increased (Figure 3A-B). Since interaction between IGFR and HER2 or HER3 contributes to trastuzumab resistance for both HR20 and pool2 cell lines (9), we next determined if ESE-1 inhibition down-regulated either of these proteins. In Pool2resistant subline, there was about a $23 \%$ decrease in HER3 protein expression although HER3 activation remain unchanged (Figure 3A-B). With regards to IGF-IR, ESE-1 inhibition resulted in reduction in both pIGF-IR protein levels in both HR20 and Pool2 cells, with the latter being due to down-regulation of IGF-IR protein (Figure 3A-B). The Pool2 cell line also exhibited a decrease in Src protein level, which largely accounted for the decrease in Src phosphorylation levels, while total Src levels in HR20 cells remain unchanged (Figure 3A-B). ESE-1 knockdown did not inhibit ERK protein levels or ERK phosphorylation in either of the cell lines, but down-regulated Akt phosphorylation at Ser473 in both cell types (Figure 3A-B). We next investigated whether ESE-1 down-regulation affects mTOR/p-mTOR, since the phosphorylation site at Ser473 is a bona fide substrate of mTORC2. ESE-1 knockdown downregulated phosphorylation of $\mathrm{mTOR}$ and $\mathrm{mTOR}$ protein 
levels in pool2 cells, indicating that regulation of mTOR, along with ERBB3, IGFR, and Src, upstream of Akt, contributes to ESE-1's mode of cell transformation, specifically in these cell types. We surmise that the increase in HER2 and HER2 phosphorylation in Pool2 cells is also because of compensatory responses arising out of prolonged mTOR inhibition. HR20 are $\mathrm{HER}^{+}$and $\mathrm{ER}^{+}$cells, and thus are considered luminal $\mathrm{B}$ breast cancer subtype, exhibited little or no changes in the level of Src or mTOR protein (Figure 3A and B) upstream of Akt. Both cell lines however evinced down-regulation of cyclin D1, a known downstream target of Akt, which most likely contributed to the growth inhibitory phenotype of ESE-1 knockdown (Figure 3A and B).

ESE-1 knockdown alone exerts an anti-proliferative effect similar to trastuzumab in parental cell lines, but does not restore sensitivity to trastuzumab in trastuzumab-resistant sublines. Given the fact that ESE-1 KD inhibited several signaling molecules that appear to function as counterregulatory pathways to trastuzumab treatment, we tested if ESE-1 knockdown sensitizes the resistant cell lines to trastuzumab-mediated growth inhibition.

Since ESE-1 knockdown results in a fairly robust inhibition of cell proliferation, we designed an experiment to test whether synergy occurs with trastuzumab plus ESE-1 knockdown by achieving a lower level of ESE-1 knockdown resulting in persistent proliferation, so that synergy could be detected. To optimize for a lower level of ESE-1 knockdown, cells were subjected to only one round of lentiviral infection and selected with puromycin. Post-selection, cells were plated in 96 wells and treated with increasing doses of trastuzumab for a length of 3 days for BT474 cells and 4 days in SKBR3 cells (Figure 4A and B). In the parental BT474 cells, trastuzumab alone caused an $18 \%, 25 \%$ and $30 \%$ decrease in cell proliferation at 10, 20 and $40 \mu \mathrm{g} / \mathrm{ml}$, respectively (Figure $4 \mathrm{~A}$, black bar). At the same doses of trastuzumab, the combination with ESE-1 knockdown resulted in 21\%, 32\%, $34 \%$ and $42 \%$ inhibition, at $0,10,20$ and $40 \mu \mathrm{g} / \mathrm{ml}$, respectively (Figure 4A, checkered bar). Thus, trastuzumab alone or ESE-1 knockdown alone achieved nearly the same level of growth inhibition (18\% vs. 21\%), and the combination is better than either treatment alone, but is neither fully additive nor synergistic. In the parental SKBR3 cells, trastuzumab alone caused a $25 \%, 36 \%$ and $39 \%$ decrease in cell proliferation at 10,20 and $40 \mu \mathrm{g} / \mathrm{ml}$, respectively (Figure 4B, black bar). The same doses of trastuzumab in combination with ESE-1 knockdown resulted in $20 \%, 36 \%, 45 \%$ and $42 \%$ inhibition, at $0,10,20$ and 40 $\mu \mathrm{g} / \mathrm{ml}$, respectively (Figure $4 \mathrm{~B}$, checkered bar). Again, showing that trastuzumab alone or ESE-1 knockdown alone achieved nearly the same level of growth inhibition (25\% vs. $20 \%$ ), and the combination is slightly better than either treatment alone, but is not synergistic.
We next investigated whether ESE-1 knockdown sensitizes trastuzumab resistant sublines to trastuzumab treatment. Compared to parental BT474 and SKBR3 cells, both HR20 and Pool2 scramble controls minimally responded to increasing doses of trastuzumab, as previously reported (9). By contrast, knocking down ESE-1 alone reduced \% survival in HR20 and Pool 2 cell lines by $58 \%$ and $25 \%$, respectively, and trastuzumab treatment failed to enhance the inhibition beyond ESE-1 knockdown alone, achieving 58-60\% inhibition in HR20 cells and 25-32\% inhibition in Pool 2 cells, at all doses of trastuzumab \pm ESE- 1 knockdown (Figures $4 \mathrm{~A}$ and 4B). These results reveal that ESE-1 knockdown alone is fairly potent inhibitor of cell proliferation in these trastuzumab-resistant cell lines, and that ESE-1 knockdown does not rescue the inhibitory trastuzumab response.

To further test for any synergistic response, a clonogenicity assay was also used to measure colony numbers in ESE-1 knockdown parental and trastuzumab-resistant cell lines treated with trastuzumab. Treatment of scramble control BT474 parental cells with trastuzumab (40 $\mu \mathrm{g} / \mathrm{ml})$ alone inhibited colony numbers by $40 \%$ at 11 days post plating (Figure 4C, black bars). ESE-1 knockdown alone caused a $44 \%$ decrease in colony numbers, and a $68 \%$ decrease in combination with $40 \mu \mathrm{g} / \mathrm{ml}$ of trastuzumab. Trastuzumabresistant HR20 cells formed many fewer colonies than the parental BT474 cells and showed a minimal response to trastuzumab treatment, whereas ESE-1 knockdown alone decreased HR 20 colony numbers by $88 \%$ did not sensitize the cells to further trastuzumab treatment (Figure 4C, grey bars). In parental SKBR3 cells, trastuzumab treatment of $40 \mu \mathrm{g} / \mathrm{ml}$ inhibited colony numbers by $43 \%$ at 11 days post plating. ESE-1 knockdown alone caused a 51\% decrease in colony numbers, and a $70 \%$ decrease in colony numbers in combination with $40 \mu \mathrm{g} / \mathrm{ml}$ of trastuzumab. Notably, trastuzumab resistant Pool 2 cells formed $31 \%$ more colonies than their parental counterparts, and showed no sensitivity to trastuzumab. Knocking down ESE-1 caused a 30\% decrease in Pool 2 colony numbers with or without trastuzumab, and as before did not sensitize the cells to trastuzumab treatment (Figure 4D, grey bars).

\section{Discussion}

Increased signaling from EGFR and IGF-IR, and deregulation of the PI3K/PTEN/Akt pathway, are key contributors to trastuzumab resistance (1). It has been long established that prolonged trastuzumab treatment leads to a compensatory increase in PI3K/Akt signaling that arises because of a Aktmediated feedback loop leading to HER3 up-regulation and heregulin-mediated HER2 up-regulation (13). Another key mediator of trastuzumab resistance is cyclin D1. In a doxycycline inducible MMTV-HER2 murine model, recurrent tumors had high expression of cyclin D1/CDK4 proteins 
A

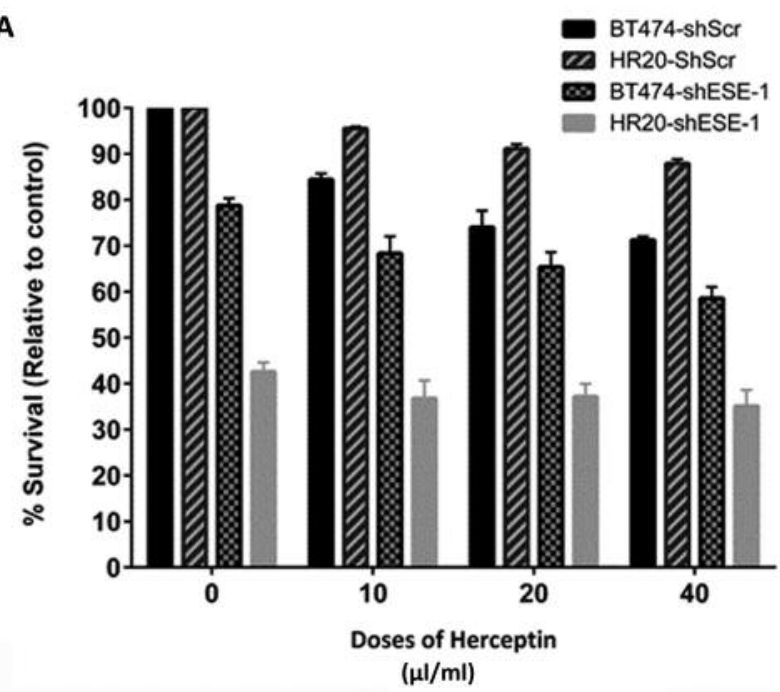

C

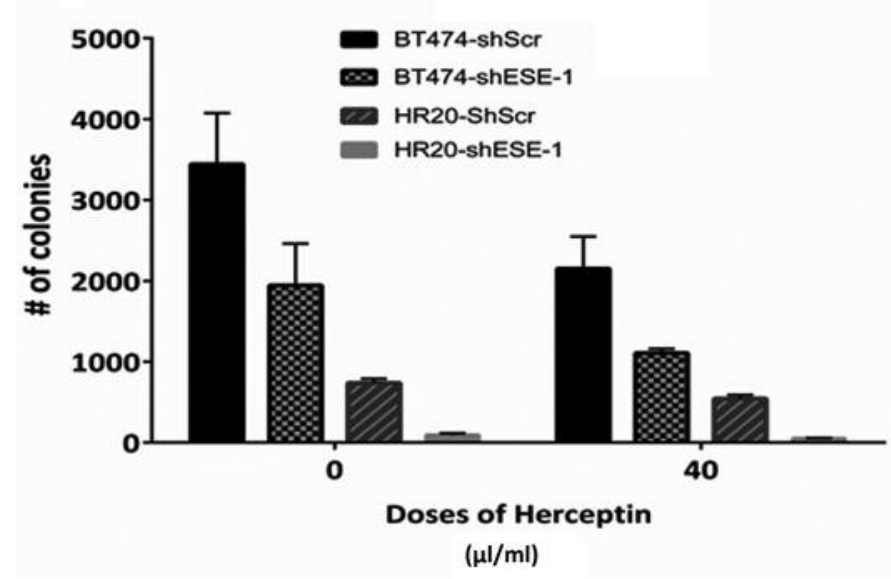

B

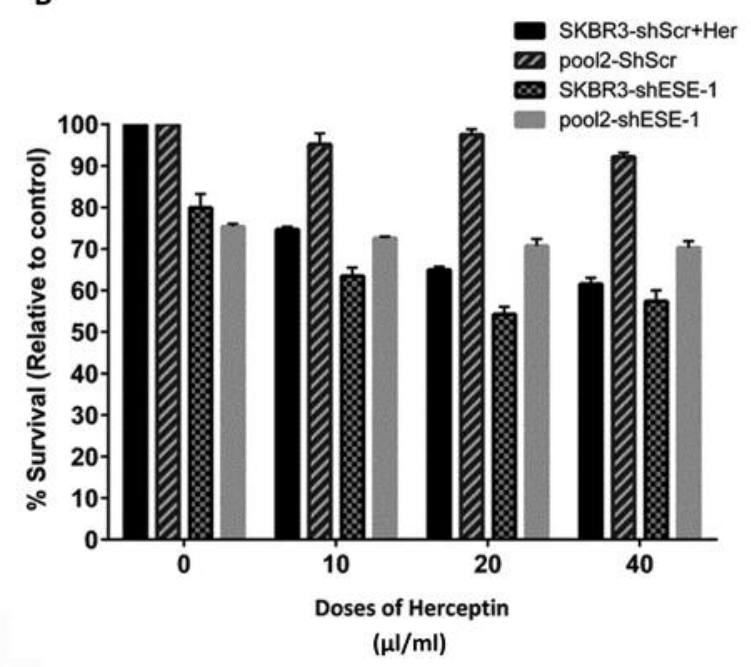

D

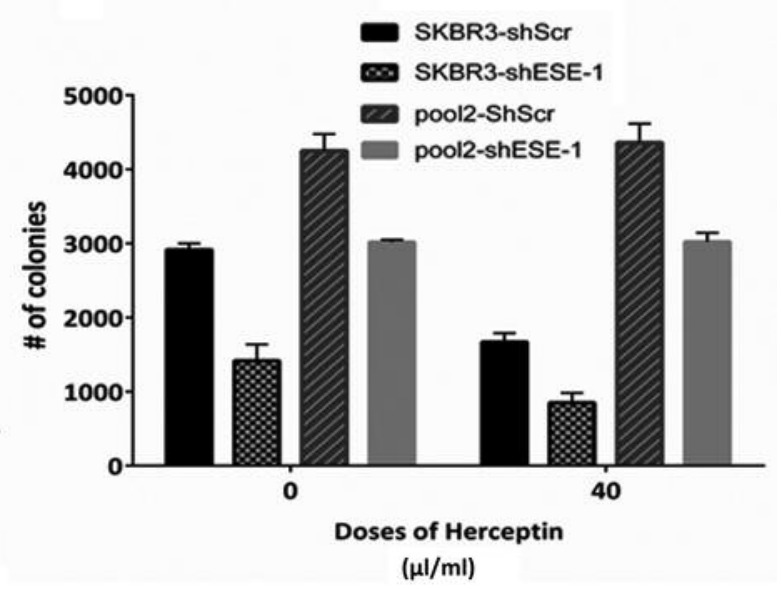

Figure 4. ESE-1 knockdown exerts trastuzumab-like anti-proliferative effects. SKBR3, BT474, Pool2 and HR20 cells were transduced with shscr or shESE-1, and were treated with increasing doses of trastuzumab or IgG control. A, B. Cells were incubated for 3 days in case of BT474 or its resistant subline HR2O and 4 days for SKBR3 and its resistant subline Pool2. Cell survival was measured using the MTS assay: Error bars are \pm SEM derived from three independent replicates. C, D: Cells were plated for clonogenicity and incubated for 11 days. Colonies were stained with crystal violet and quantified using the Image $J$ software. Error bars are \pm SEM derived from three replicates.

resulting in cyclin D1/CDK4-mediated resistance to targeted therapy for HER2 ${ }^{+}$. This was overcome using CDK4/6 inhibitors $(14,15)$. Thus, a key gap is to identify targets that can overcome trastuzumab resistance. Here we show that knocking down ESE-1 in two distinct trastuzumab-resistant cell lines, HR20 and Pool2, resulted in a significant reduction in HR20 and Pool2 cell number, cell growth, colony formation and anchorage independent growth. Thus, ESE-1 and its key downstream effectors, serve as novel targets in the treatment of trastuzumab-resistant HER2 breast cancer.

Using cell culture models of trastuzumab resistance we show that ESE-1 knockdown in the resistant sublines have a cell type-specific effect on HER2 signaling and other protein kinases, such as HER3, IGF-1R, Src and mTOR. In the trastuzumab-resistant HR20 cells, which are $\mathrm{ER}^{+}$, HER2 ${ }^{+}$ luminal B cells, ESE-1 inhibition decreases HER2 protein expression, HER2 phosphorylation. It appears that in trastuzumab-resistant SKBR3/pool2 cell lines, the antiproliferative effects of ESE-1 knockdown are driven by inhibition in expression levels of mTOR, Src, and IGF-IR, and not by changes in phosphorylation of their respective proteins. In both resistant sublines, however, there is downstream inhibition in Akt activation, and a decrease in downstream cyclin D1. This reveals that ESE-1 is able to dictate cell growth responses by controlling key transformation nodes, such as Akt, which functions upstream 
of the cell cycle, and also by controlling cyclin D, which directly regulates the cell cycle.

While it is known that ESE-1 is a transcriptional activator of HER2 $(6,8)$, and EHF (an ETS transcription factor) transcriptionally regulates HER3 in thyroid cells (16), further studies are required to understand how ESE-1 controls expression of specific proteins, such as HER3, mTOR, IGFR, and Src in the trastuzumab-resistant sublines. Whole genome analysis in ESE-1 knockdown parental SKBR3 cells does not show a decrease in the level of mTOR, Src, or IFG-1R mRNA, suggesting that they are unlikely to be direct transcriptional targets of ESE-1 and are indirectly modulated by ESE-1 to control transformation properties in the resistant sublines.

The fact that ESE-1 knockdown inhibits mTOR and Src expression and activation has special implications in trastuzumab therapy. The baseline Src activation observed in the resistant sublines, especially in Pool2, corroborates with previous findings, which showed that Src activation following IGFR phosphorylation is a compensatory response in these particular cell types (9). Up-regulation in Src or mTOR expression leading to aberrant Akt signaling is one of many mechanisms of lapatinib resistance $(17,18)$. In preclinical models of trastuzumab resistance, use of mTOR inhibitors has resulted in enhanced anti-proliferative effects, and there is some clinical evidence for the utility of mTOR inhibition in hormone receptor-positive and HER2-positive breast cancers (19-21).

We, therefore, tested if ESE-1 knockdown enhanced trastuzumab mediated growth inhibition in the parental cell lines or if it sensitized the resistant cells to trastuzumab treatment. Although ESE-1 knockdown did not enhance trastuzumab sensitivity in either case, it alone had a pronounced inhibitory effect on cell survival and colony growth in both parental BT474 and SKBR3 cell lines and trastuzumab-resistant derivative HR20 and pool 2 cell lines. This was not surprising given that ESE-1 controls the transformation properties of the parental cells via PI3K/Akt activation (7), a pathway that is commonly targeted by trastuzumab. One other mechanism by which trastuzumab controls growth response is via antibodydependent cell cytotoxicity and apoptosis. ESE-1 knockdown had no effect on apoptosis and did not sensitize trastuzumab resistant cell for apoptosis. Nonetheless, collectively these studies established that ESE-1 modulates several signaling molecules, that commonly contribute to HER inhibitor resistance and therefore provides a rationale for investigating the signaling pathways of ESE-1 in HER2 ${ }^{+}$patients showing resistance to anti HER2 therapy.

\section{Acknowledgements}

The Authors would like to acknowledge contributions of every Author involved in the preparation of the manuscript.

\section{References}

1 Luque-Cabal M, Garcia-Teijido P, Fernandez-Perez Y, SanchezLorenzo L and Palacio-Vazquez I: Mechanisms Behind the Resistance to Trastuzumab in HER2-Amplified Breast Cancer and Strategies to Overcome It. Clin Med Insights Oncol 10: 2130, 2016.

2 Molina MA, Codony-Servat J, Albanell J, Rojo F, Arribas J and Baselga J: Trastuzumab (herceptin), a humanized anti-Her2 receptor monoclonal antibody, inhibits basal and activated Her2 ectodomain cleavage in breast cancer cells. Cancer Res 61: 4744-4749, 2001.

3 Maximiano S, Magalhaes P, Guerreiro MP and Morgado M: Trastuzumab in the Treatment of Breast Cancer. BioDrugs 30: 75-86, 2016.

4 Dahabreh IJ, Linardou H, Siannis F, Fountzilas G and Murray $\mathrm{S}$ : Trastuzumab in the adjuvant treatment of early-stage breast cancer: a systematic review and meta-analysis of randomized controlled trials. Oncologist 13: 620-630, 2008.

5 Madrid-Paredes A, Canadas-Garre M, Sanchez-Pozo A and Calleja-Hernandez MA: Non-HER2 signaling pathways activated in resistance to anti-HER2 therapy in breast cancer. Breast Cancer Res 153: 493-505, 2015.

6 Eckel KL, Tentler JJ, Cappetta GJ, Diamond SE and GutierrezHartmann A: The epithelial-specific ETS transcription factor ESX/ESE-1/Elf-3 modulates breast cancer-associated gene expression. DNA Cell Biol 22: 79-94, 2003.

7 Kar A and Gutierrez-Hartmann A: ESE-1/ELF3 mRNA expression associates with poor survival outcomes in HER2 ${ }^{+}$ breast cancer patients and is critical for tumorigenesis in HER2+ breast cancer cells. Oncotarget 8: 69622-69640, 2017.

8 Neve RM, Ylstra B, Chang CH, Albertson DG and Benz CC: ErbB2 activation of ESX gene expression. Oncogene 21: 39343938, 2002.

9 Huang X, Gao L, Wang S, McManaman JL, Thor AD, Yang X, Esteva FJ and Liu B: Heterotrimerization of the growth factor receptors erbB2, erbB3, and insulin-like growth factor-i receptor in breast cancer cells resistant to herceptin. Cancer Res 70: 1204-1214, 2010.

10 Prescott JD, Poczobutt JM, Tentler JJ, Walker DM and Gutierrez-Hartmann A: Mapping of ESE-1 subdomains required to initiate mammary epithelial cell transformation via a cytoplasmic mechanism. Mol Cancer 10: 103, 2011.

11 Nahta R, Takahashi T, Ueno NT, Hung MC and Esteva FJ: P27(kip1) down-regulation is associated with trastuzumab resistance in breast cancer cells. Cancer Res 64: 3981-3986, 2004.

12 Huang J, Wang S, Lyu H, Cai B, Yang X, Wang J and Liu B: The anti-erbB3 antibody MM-121/SAR256212 in combination with trastuzumab exerts potent antitumor activity against trastuzumabresistant breast cancer cells. Mol Cancer 12: 134, 2013.

13 Gijsen M, King P, Perera T, Parker PJ, Harris AL, Larijani B and Kong A: HER2 phosphorylation is maintained by a PKB negative feedback loop in response to anti-HER2 herceptin in breast cancer. PLoS Biol 8: e1000563, 2010.

14 Goel S, Wang Q, Watt AC, Tolaney SM, Dillon DA, Li W, Ramm S, Palmer AC, Yuzugullu H, Varadan V, Tuck D, Harris LN, Wong KK, Liu XS, Sicinski P, Winer EP, Krop IE and Zhao JJ: Overcoming Therapeutic Resistance in HER2-Positive Breast Cancers with CDK4/6 Inhibitors. Cancer Cell 29: 255-269, 2016. 
15 Witkiewicz AK, Cox D and Knudsen ES: CDK4/6 inhibition provides a potent adjunct to Her2-targeted therapies in preclinical breast cancer models. Genes Cancer 5: 261-272, 2014

16 Lv Y, Sui F, Ma J, Ren X, Yang Q, Zhang Y, Guan H, Shi B, Hou $\mathrm{P}$ and Ji M: Increased expression of EHF contributes to thyroid tumorigenesis through transcriptionally regulating HER2 and HER3. Oncotarget 7: 57978-57990, 2016.

17 Brady SW, Zhang J, Tsai MH and Yu D: PI3K-independent mTOR activation promotes lapatinib resistance and IAP expression that can be effectively reversed by mTOR and Hsp90 inhibition. Cancer Biol Ther 16: 402-411, 2015.

18 Formisano L, Nappi L, Rosa R, Marciano R, D'Amato C, D'Amato V, Damiano V, Raimondo L, Iommelli F, Scorziello A, Troncone G, Veneziani B, Parsons SJ, De Placido S and Bianco $\mathrm{R}$ : Epidermal growth factor-receptor activation modulates Srcdependent resistance to lapatinib in breast cancer models. Breast Cancer Res 16: R45, 2014.

19 O’Brien NA, McDonald K, Tong L, von Euw E, Kalous O, Conklin D, Hurvitz SA, di Tomaso E, Schnell C, Linnartz R, Finn RS, Hirawat $S$ and Slamon DJ: Targeting PI3K/mTOR overcomes resistance to HER2-targeted therapy independent of feedback activation of AKT. CClin Cancer Res 20: 3507-3520, 2014.
20 Hurvitz SA, Andre F, Jiang Z, Shao Z, Mano MS, Neciosup SP, Tseng LM, Zhang Q, Shen K, Liu D, Dreosti LM, Burris HA, Toi M, Buyse ME, Cabaribere D, Lindsay MA, Rao S, Pacaud LB, Taran T and Slamon D: Combination of everolimus with trastuzumab plus paclitaxel as first-line treatment for patients with HER2-positive advanced breast cancer (BOLERO-1): a phase 3, randomised, double-blind, multicentre trial. Lancet Oncol 16: 816-829, 2015.

21 Andre F, O’Regan R, Ozguroglu M, Toi M, Xu B, Jerusalem G, Masuda N, Wilks S, Arena F, Isaacs C, Yap YS, Papai Z, Lang I, Armstrong A, Lerzo G, White M, Shen K, Litton J, Chen D, Zhang Y, Ali S, Taran T and Gianni L: Everolimus for women with trastuzumab-resistant, HER2-positive, advanced breast cancer (BOLERO-3): a randomised, double-blind, placebocontrolled phase 3 trial. Lancet Oncol 15: 580-591, 2014.

Received August 22, 2017

Revised September 25, 2017

Accepted September 29, 2017 